\title{
NEW APPROACH TO OPTICAL POLARISATION MODE DISPERSION MITIGATION: EXPERIMENTAL ANALYSIS OF THE DYNAMIC PERFORMANCES OF A COST-DRIVEN DEVICE
}

\author{
Raoul Fiorone, Aldo Perasso, Massimo Speciale, Marco Camera, Andrea Corti \\ MarconiCommunications, Via A. Negrone, 1/A-16153 Genova, Italy \\ raoul.fiorone@marconi.com, aldo.perasso@marconi.com, massimo.speciale@marconi.com, \\ marco.camera@maconi.com, andrea.corti@marconi.com
}

\begin{abstract}
An innovative approach to optical mitigation is proposed. We experimentally investigate the dynamic performance of a simple Polarisation Mode Dispersion optical mitigator, and demonstrate that a cost-effective solution can minimise the real criticality of optical structures.
\end{abstract}

\section{INTRODUCTION}

Polarisation Mode Dispersion (PMD) can be the major limiting factor for future $40 \mathrm{Gbit} / \mathrm{s}$ optical systems [1] and can constitute a headache in case of $10 \mathrm{Gbit} / \mathrm{s}$ all-optical networks. An intense activity has been carried out in the last years by the scientific community [2], but to date no real solution to mitigate the effects of PMD is available for practical deployment. The development of a PMD compensator (PMDC) is not a usual engineering problem, given the high number of non-independent variables, the statistical behaviour of the physical phenomena, and the economical and technological constraints related to a practical use.

This paper describes the design and characterisation work behind the development of a PMDC, and analyses the behaviour of a cost-driven single-stage 
device, starting from where other solutions have failed so far, both cost-wise and performance-wise.

The performances of a simple, cost-effective and reliable optical PMDC come out to be quite attractive, even without possible supplementary aids like Forward Error Correction (FEC), adaptive Clock and Data Recovery and electronic equalisation.

\section{THE DESIGN OF EXPERIMENT}

A bunch of polarisation controllers (PC) and some metres of polarisation maintaining fibre can be easily used to open a PMD-affected eye diagram. However, it is not immediate to figure out the subtle troubles that the design of an optical PMDC involves. Our first hand experience has taught us that without a systematic Design of Experiment (DOE) approach $[3,4]$ and properly chosen lab equipment, the chances to find the right recipe to design a good (cost sensible, effective and reliable) optical PMDC are very low [5].

A DOE is a structured, organised method to identify and determine the mutual relationship among the factors affecting a complex process. It is a sort of mathematical procedure to span the entire operating domain with a minimum set of test cases.

\section{THE "COMPENSATION MODE DILEMMA"}

The impairments due to PMD depend upon the signal's State of Polarisation (SOP) and the fibre parameters, Differential Group Delay (DGD) and Principal States of Polarisation (PSP) in a 1st order analysis. Given the statistical behaviour of all the parameters [6], the Outage Probability (OP) concept [7] is needed to evaluate the system performances, both without and with PMDC [8].

The obvious expectation is a compensated system showing an essentially lower OP on a PMD affected link, so that a known margin, whose probability to be exceeded over time is equal to the OP, can be allocated in the system design.

The OP is usually calculated and checked emulating the real world dynamic behaviour with a sequence of (quasi) static conditions. The two cases do not exactly coincide, and different behaviours can unsurprisingly be expected. In case of real dynamic characterisation of an optical PMDC, it is possible to find out that the transients from one working condition to the next are not always hitless, as one could expect [8]. A few solutions have been proposed with other rationales to constrain the PMDC operation mode, with the result of adding extra cost and complexity [9]. 
The assumption that, given a PC endless operation, for every continuous evolution of the signal SOP and fibre PMD, the optimum PMDC parameters evolve continuously, is not necessarily true, and in our DOE-like analysis we have, for the first time, experimentally observed and explained this potentially dangerous phenomenon. To do so, it is necessary to perform accurate PMDC state monitoring, long-term logging, and Bit Error Ratio (BER) floor and burst detection and monitoring, in order to identify, unequivocally recognise and finally understand the killer events.
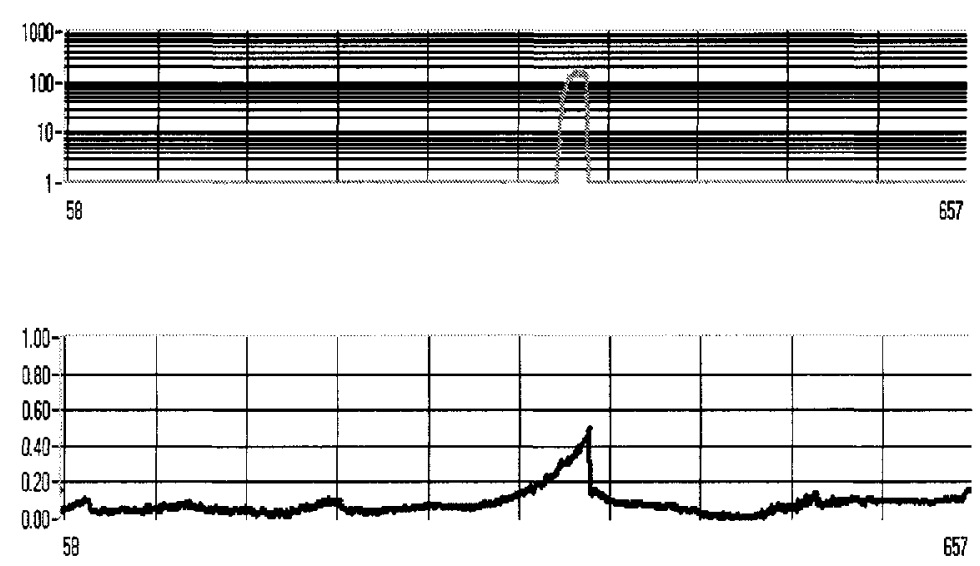

Figure 1. Experimental evidence of the "compensation mode dilemma" phenomenon

In Figure 1 the experimental evidence of the occurrence of the "compensation mode dilemma" is presented. What is important to notice is that the error burst occurred (error graph - top) without any reset condition of the PC's and with the feedback signal well aware of the problem (mean square error (MSE) graph bottom). In this experiment the feedback signal was based on a conventional RF spectrum monitoring, and the PMDC was a two-section optical structure, with ample capability to mitigate the PMD values emulated.

The OP is not enough to fully describe the PMDC performances, and the reliability of the whole system, but it can usefully provide an upper bound limit. 


\section{THE OBJECTIVE}

Our objective was to adopt a cost and integration driven approach to build a simple 1st order PMDC.

It is curious to notice that the best performing PMDC that we have tested in our lab (not completely exempt from the "compensation mode dilemma", anyhow) was too big and expensive for real deployment: it was a performance-driven device.

Our choice has been a single-stage optical PMDC, using RF spectrum based PMD monitoring. The target was to mitigate up to $0.6 \div 0.8$ Unit Interval peak DGD with real world polarisation variations, which are normally slower than those emulated (up to Scan Rate (SR) 4 of the Agilent 11896A PC's used with the PMD Emulator - PMDE). No "compensation mode dilemma" criticality was a design objective and considered a real differentiator.

\section{PERFORMANCE ANALYSIS}

The design of the PMDC has been driven by the ability to fully assess the device's behaviour using a DOE-like approach and a programmable PMDE. The performances have been investigated to check, in the presence of different Optical Signal to Noise Ratio (OSNR) and Polarisation Dependent Loss values: compensation capability, dynamic behaviour in terms of response speed, endless operation, BER floor and "compensation mode dilemma".

In Figure 2 the sketch of the test bed is given.

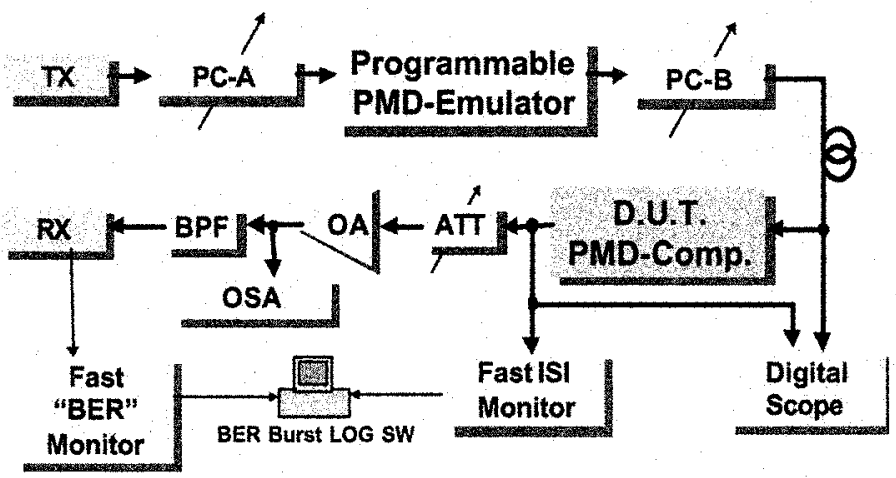

Figure 2. The test bed

In Figure 3 the system tolerance against DGD is shown in case of $10 \mathrm{Gbit} / \mathrm{s}$ NRZ signal, with $19 \mathrm{~dB}$ OSNR, no FEC, linear operation and practically no chromatic dispersion. Using a $2 \mathrm{~dB}$ OSNR penalty threshold, the system resistance 
against DGD (and SOP/PSP scanning) results increased from 30ps to more than 80 ps, nearly a three-fold improvement.

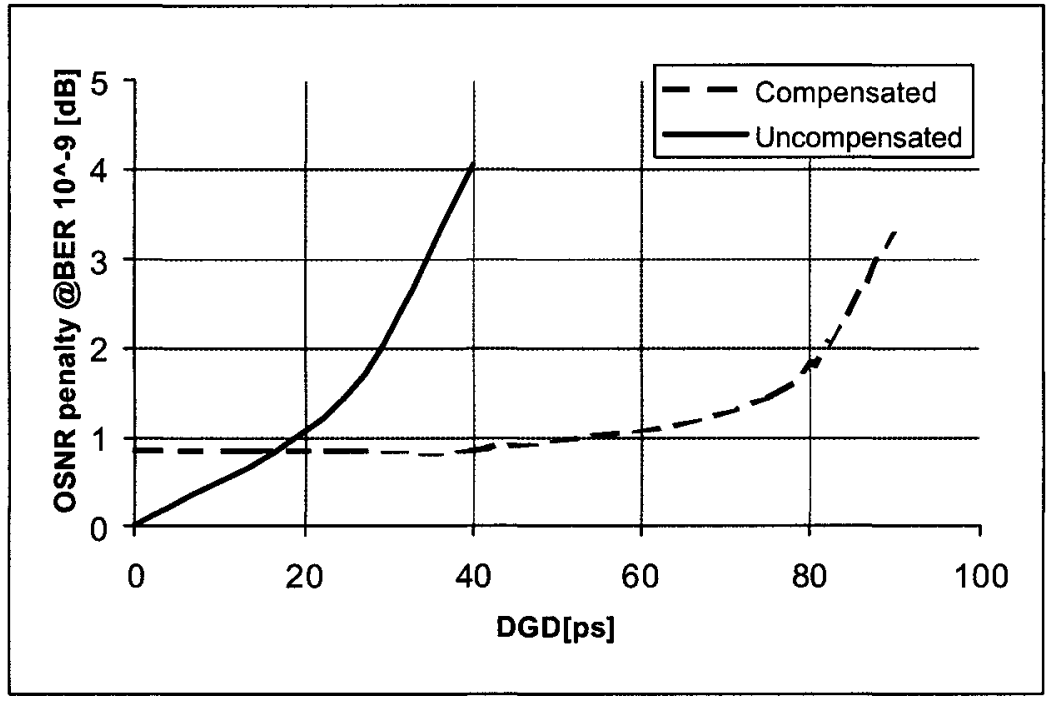

Figure 3. Long-term performance results against DGD

In our preliminary results, we have found no BER floor and no "compensation mode dilemma" events in a series of test sessions long at least 36 hours (SR 2 for PC-A and PC-B, Figure 2). It is worth noting that, in the same dynamic conditions, other PMDC prototypes have shown "compensation mode dilemma" events in a few minutes (typically $10 \div 30$ ).

\section{CONCLUSIONS}

A highly innovative approach has allowed us to achieve for the first time a really complete assessment of the performances of an optical PMDC. We have demonstrated that the only way to design a deployable PMDC is a DOE-like method, with both static and dynamic comprehensive investigations.

The cost-driven single-stage PMDC that we have designed and fully characterised, in case of FEC-free 10Gbit/s NRZ signal, has shown efficacy up to more than 80ps DGD, with fast tracking capability, no error burst events and no BER floor. 


\section{REFERENCES}

[1] H. Sunnerud, M. Karlsson, C. Xie, P.A. Andrekson, "Polarization Mode Dispersion in High Speed Fiber-Optic Transmission Systems" J. Lightwave Technol., 20, Dec 2002, pp. 2204-2219.

[2] Proceedings of Venice Summer School on PMD, June 2002

[3] R. A. Fisher, "The design of experiments" (9th ed.), 1971, New York, Hafner Publishing Co.

[4] J. J. Kennedy, A.J. Bush, "An introduction to the design and analysis of experiments", Lanham, MD, University Press of America Inc.

[5] A. Perasso, R. Fiorone, A. Corti, ECOC2003 Workshop on "PMD: Causes, Effects and Cures".

[6] F. Curti, B. Daino, G. De Marchis, and F. Matera, "Statistical treatment of the evolution of the principal states of polarization in single-mode fibers J. Lightwave Technol., vol. 8, 1990, pp.1162-1166.

[7] H. Bulow, "System outage probability due to first and second order PMD" IEEE Photon. Technol. Lett. Oct. 1998, pp. 696-699

[8] E. Ciaramella, "Theoretical Evidence of Dynamical Limitations in Practical Singlestage PMD Compensators", submitted to IEEE Photon. Technol. Lett.

[9] H. Y. Pua, K. Peddanarppagari, B. Zhu, C. Allen, K. Demarest and R. Hui, "An adaptive First order Polarization Mode Dispersion compensation system aided by polarization scrambling: theory and demonstration", J. Lightwave Technol, vol. 18, June 2000, pp. 832-841 